\begin{tabular}{|c|l|}
\hline Title & Hydration of alkali ions from first principles molecular dynamics revisited \\
\hline Author(s) & Ikeda, Takashi; Boero, Mauro; Terakura, Kiyoyuki \\
\hline Citation & $\begin{array}{l}\text { The Journal of Chemical Physics, 126(3), 034501 } \\
\text { https://doi.org/10.1063/1.2424710 }\end{array}$ \\
\hline Issue Date & 2007-01-21 \\
\hline Doc URL & http://hdl.handle.net/2115/18644 \\
\hline Rights & Copyright $\odot 2007$ American Institute of Physics \\
\hline Type & article \\
\hline File Information & JCP126-3.pdf \\
\hline
\end{tabular}

Instructions for use 


\title{
Hydration of alkali ions from first principles molecular dynamics revisited
}

\author{
Takashi Ikeda \\ Synchrotron Radiation Research Unit, Quantum Beam Science Directorate (QuBS), Japan Atomic Energy \\ Agency (JAEA), 1-1-1 Kouto, Sayo, Hyogo 679-5148, Japan \\ Mauro Boero \\ Center for Computational Sciences, University of Tsukuba, 1-1-1 Tennodai, Ibaraki 305-8577, Japan \\ and CREST, Japan Science and Technology Agency, 4-1-8 Honcho, Kawaguchi, Saitama 332-0012, Japan
}

\author{
Kiyoyuki Terakura \\ Division of Frontier Research, Creative Research Initiative "Sousei," Hokkaido University, Kita 21, \\ Nishi 10, Sapporo 001-0021, Japan
}

(Received 16 October 2006; accepted 29 November 2006; published online 16 January 2007)

\begin{abstract}
Structural and dynamical properties of the hydration of $\mathrm{Li}^{+}, \mathrm{Na}^{+}$, and $\mathrm{K}^{+}$in liquid water at ambient conditions were studied by first principles molecular dynamics. Our simulations successfully captured the different hydration behavior shown by the three alkali ions as observed in experiments. The present analyses of the dependence of the self-diffusion coefficient and rotational correlation time of water on the ion concentration suggest that $\mathrm{Li}^{+}\left(\mathrm{K}^{+}\right)$is certainly categorized as a structure maker (breaker), whereas $\mathrm{Na}^{+}$acts as a weak structure breaker. An analysis of the relevant electronic structures, based on maximally localized Wannier functions, revealed that the dipole moment of $\mathrm{H}_{2} \mathrm{O}$ molecules in the first solvation shell of $\mathrm{Na}^{+}$and $\mathrm{K}^{+}$decreases by about $0.1 \mathrm{D}$ compared to that in the bulk, due to a contraction of the oxygen lone pair orbital pointing toward the metal ion. (C) 2007 American Institute of Physics. [DOI: 10.1063/1.2424710]
\end{abstract}

\section{INTRODUCTION}

The importance of the hydration of metal ions stems from its fundamental role in a wide variety of chemical and biological systems. This issue dates back to the early 1930s, and since then a wide range of experiments have been performed by utilizing elaborate techniques to unravel the most important points, such as ion-water distance, hydration structure, dynamical properties of hydrated ions, etc. Such a wealth of knowledge can be found in well assessed monographs ${ }^{1-4}$ and review articles ${ }^{5,6}$ in major scientific journals.

In particular, alkali ions deserve special attention, especially sodium and potassium, since they are abundant in natural waters and play a special role in biochemistry. The concentration of $\mathrm{Na}^{+}$and $\mathrm{K}^{+}$in sea water amounts to about 460 and $10 \mathrm{mM}$, respectively. ${ }^{7}$ Analogously, the concentration of $\mathrm{Na}^{+}$and $\mathrm{K}^{+}$outside a mammalian blood cell is very close to what is found in sea water, whereas their concentrations are, in a rough approximation, reversed inside the cell. Due to these peculiarities, sodium and potassium ions are classified as major components of the human (and mammals) body, constituting about $1 \%$ of our weight, along with $\mathrm{Mg}$ and $\mathrm{Ca}$ ions.

Despite decades of efforts, the microscopic details concerning the solvation shells of these ions still escape accurate experimental investigations. Yet, in view of the emerging field of nanobiotechnology, their detailed atomistic picture is of fundamental importance. Experimental uncertainties in determining the hydration structure of $\mathrm{Na}^{+}$and $\mathrm{K}^{+}$are due to a number of reasons. One of these reasons is the ion-water distance, which is very close to the oxygen-oxygen distance in bulk water, especially for $\mathrm{K}^{+}$. This makes it very difficult to separate structure factors and pair correlation functions, as provided by x-ray and neutron diffraction experiments, of the different species. Another difficulty is represented by the inherent flexibility of the solvation shells of both ions. Water exchange is likely to occur between the first and second solvation shells at the rate of a few picoseconds or less. This indicates that the solvation shells of $\mathrm{Na}^{+}$and $\mathrm{K}^{+}$are not well defined, contrary, for instance, to the rather rigid hydration shell of $\mathrm{Li}^{+}$, whose experimental investigation is much less problematic.

In principle, molecular dynamics (MD) simulations can help to fill the gap represented by these experimental limitations and could provide an atomistic insight into the structure and dynamics of the solvation shell. In this respect, first principles MD, proposed by Car and Parrinello more than 20 years ago, ${ }^{8}$ is a well assessed method nowadays routinely used to complement experimental data and to predict a variety of physical and chemical properties (see, e.g., Ref. 9 and references therein). The major advantages rely on the fact that this approach is particularly suited for investigating fast phenomena accompanied by changes in the electronic structure; it does not require any predefined force fields but forces acting on the atoms are computed on the fly from quantum mechanics within the density functional theory (DFT) formulation. $^{10}$

Since the pioneering first principles MD for the solvated $\mathrm{Be}^{2+}$ in water was reported, ${ }^{11}$ its application for ionic hydration has been extended to many metal ions including $\mathrm{Mg}^{2+}$ (Ref. 12), $\mathrm{Al}^{3+}$ (Ref. 13), $\mathrm{Ca}^{2+}$ (Ref. 14), etc. Former first principles MD simulations for $\mathrm{Li}^{+}$(Ref. 15), $\mathrm{Na}^{+}$(Refs. 16 
and 17), and $\mathrm{K}^{+}$(Ref. 18) successfully described the structure of first solvation shell of these alkali ions and also observed several water-exchange processes for $\mathrm{Na}^{+}$and $\mathrm{K}^{+}$in a simulation time of $\sim 2 \mathrm{ps}$. However, such a very short time, affecting all previous simulations, prevented any estimate of dynamical quantities. Indeed, this is an issue that has been extensively discussed in recent works ${ }^{19-23}$ and that is now rather clear to the scientific community. Recent advancement in computer resources allows nowadays to perform simulations for a much longer time scale, namely one order of magnitude longer, than the past. This, in turn, enables us to estimate dynamical quantities such as self-diffusion coefficient, rotational correlation time, etc., at least at a semiquantitative level.

In this work we studied systematically the structure of the hydration shells of $\mathrm{Li}^{+}, \mathrm{Na}^{+}$, and $\mathrm{K}^{+}$using first principles MD. The self-diffusion coefficient and rotational correlation time of water molecules in aqueous solutions of the three alkali ions were then estimated to examine their dependence on the ion concentration, which is known to be peculiar to each ionic species. In particular, we focus on the relationship among molecular orientation, formation of hydrogen bonds, and polarization of water molecules. These features allow for a detailed inspection of the differences in the hydration of the three alkali ions.

The remainder of the paper is organized as follows. In Sec. II we describe the computational method including some technical details relevant to the present study. In Sec. III the structural properties of the first solvation shell and dynamics of water molecules in the aqueous solutions are discussed in detail and supported by an analysis of the polarization effect on hydration water molecules. Finally, in Sec. IV we draw some conclusions.

\section{COMPUTATIONAL DETAILS}

We performed Car-Parrinello ${ }^{8} \mathrm{MD}$ simulations of alkali ions in liquid water at ordinary density $\left(1.0 \mathrm{~g} / \mathrm{cm}^{3}\right)$ and temperature $(T=300 \mathrm{~K})$ within $\mathrm{DFT},{ }^{10}$ including gradient corrections to the exchange-correlation functional after Hamprecht-Cohen-Tozer-Handy ${ }^{24}$ (HCTH). The performance of this particular functional for liquid water and solution systems has already been investigated and assessed in previous works. ${ }^{25-27}$ The valence-core interaction was described by norm-conserving Troullier-Martins pseudopotentials $^{28}$ for $\mathrm{Na}, \mathrm{K}$, and $\mathrm{O}$, a Goedecker-TeterHutter type pseudopotential ${ }^{29,30}$ for $\mathrm{Li}$, and a von Barth-Car analytical pseudopotential ${ }^{31}$ for $\mathrm{H}$. The pseudopotentials for $\mathrm{Na}$ and $\mathrm{K}$ were generated by including semicore states; the $2 s$ and $2 p$ semicore states of a sodium ion were treated as valence states and the core radius of $1.0 \AA$ was used for pseudization of both the $s$ and $p$ channels. For potassium, the $3 s$ and $3 p$ states were also treated as valence states; core radii of $1.2,1.2$, and $1.5 \AA$ were used for pseudization of $s$, $p$, and $d$ channels, respectively. Valence orbitals were expanded in a plane wave (PW) basis set with an energy cutoff of 80 Ry. Periodic boundary conditions on the simulation cell were applied and the Brillouin zone was sampled at the $\Gamma$ point only. The equations of motion were integrated with a
TABLE I. Validation of pseudopotential-based PW-DFT approach for the aqueous alkali ion complexes $\mathrm{M}^{+} \mathrm{H}_{2} \mathrm{O}(\mathrm{M}=\mathrm{Li}, \mathrm{Na}$, and $\mathrm{K})$. Cation-oxygen distances $d$ are given in $\AA$ and hydration energies $\Delta E$ in $\mathrm{kcal} / \mathrm{mol}$. PW-DFT results agree excellently with quantum chemistry quantities computed at the MP2/6-311+ $+\mathrm{G}(d, p)$ level of theory (Ref. 32).

\begin{tabular}{lccc}
\hline \hline System & Quantity & G03 (MP2/6-311++G(d,p)) & CPMD (80 Ry) \\
\hline $\mathrm{Li}^{+} \mathrm{H}_{2} \mathrm{O}$ & $d$ & 1.879 & 1.882 \\
& $\Delta E$ & 33.4 & 33.6 \\
$\mathrm{Na}^{+} \mathrm{H}_{2} \mathrm{O}$ & $d$ & 2.292 & 2.244 \\
& $\Delta E$ & 23.1 & 23.8 \\
$\mathrm{~K}^{+} \mathrm{H}_{2} \mathrm{O}$ & $d$ & 2.669 & 2.661 \\
& $\Delta E$ & 17.9 & 16.9 \\
\hline \hline
\end{tabular}

time step of 4 a.u. (0.0968 fs) using a fictitious electron mass of 400 a.u.; these values of the simulation parameters have already been shown to be crucial to obtain reliable static and dynamical properties ${ }^{19,20}$ and to keep Car-Parrinello trajectories close to the Born-Oppenheimer surface. ${ }^{33}$ Our pseudopotential-based PW-DFT approach was validated by auxiliary all-electron calculations in order to provide a careful check of the ion-oxygen distances $d$ and related hydration energies $\Delta E$ for $\mathrm{M}^{+} \mathrm{H}_{2} \mathrm{O}$ complexes $(\mathrm{M}=\mathrm{Li}, \mathrm{Na}$, and $\mathrm{K})$. As shown in Table I, the PW-DFT results agree excellently with the corresponding estimate made with a standard quantum chemistry method at the MP2/6-311++G(d,p) level of theory. ${ }^{32}$

The dependence of dynamical quantities upon the concentration of the metal ions was examined by performing the two different simulations for each metal species. The first set of simulations were performed on systems consisting of one metal ion plus 63 water molecules in a periodically repeated cubic box of side $L=12.417 \AA$. The second set of calculations, instead, were done for an identical simulation cell containing two metal ions and 62 water molecules; namely, one of the solvent $\mathrm{H}_{2} \mathrm{O}$ molecules was replaced by a second metal ion, thus doubling the ion concentration. Note that this replacement leads to the systematic decrease of the computed pressure with respect to the first set by $190 \mathrm{MPa}$ in the case of $\mathrm{Li}^{+}$to $90 \mathrm{MPa}$ in the case of $\mathrm{K}^{+}$. We remark, however, that this decrease of the computed pressure is accompanied by the large fluctuations of $\sim 300 \mathrm{MPa}$ due presumably to the small system size. Therefore, the changes in pressure we estimated are on the verge of the accuracy of present PWDFT calculations. ${ }^{34}$ The excess charge of both samples was compensated by a uniform background charge instead of adding a counterion explicitly. After equilibrating the system at $300 \mathrm{~K}$ for about $2 \mathrm{ps}$ the trajectories were collected for 40 ps. All the simulations were done on a $(N, V, T)$ canonical ensemble in which the temperature $(T=300 \mathrm{~K})$ was controlled by a Nosé-Hoover thermostat ${ }^{35,36}$ and performed with the CPMD package. ${ }^{37}$

\section{RESULTS AND DISCUSSION}

\section{A. Structural properties}

One of the basic questions relevant to the characterization of the hydration of a metal ion is the (average) ion-water distance. This information is extracted both experimentally and computationally from the radial distribution functions 

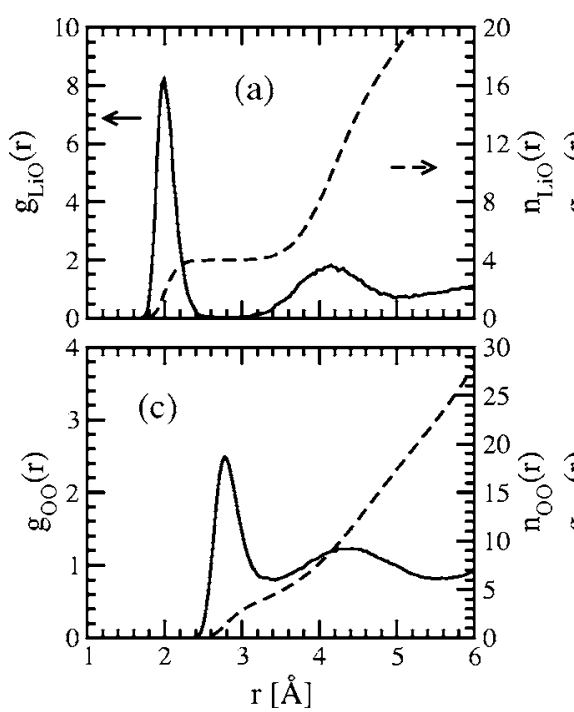
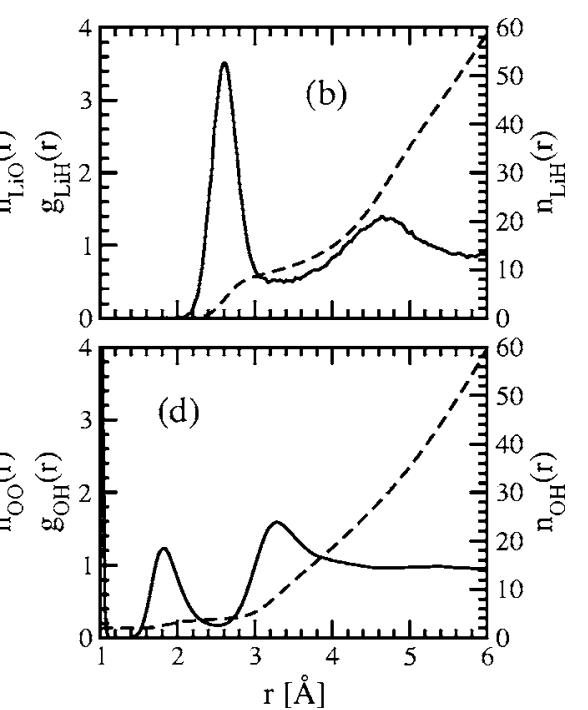

FIG. 1. Radial distribution functions (a) $g_{\mathrm{LiO}}(r)$, (b) $g_{\mathrm{LiH}}(r),(\mathrm{c}) g_{\mathrm{OO}}(r)$, and (d) $g_{\mathrm{OH}}(r)$ for $\mathrm{Li}^{+}$in liquid water. The dashed line in each panel represents the corresponding running coordination number $n(r)$.
(RDF). For the case of $\mathrm{Li}^{+}$, the outcome of our simulation is summarized in Fig. 1 where the RDFs $g_{\mathrm{LiO}}(r), g_{\mathrm{LiH}}(r)$, $g_{\mathrm{OO}}(r)$, and $g_{\mathrm{OH}}(r)$ are shown. The first peak of $g_{\mathrm{LiO}}(r)$ is located at $1.99 \AA$, about $0.1 \AA$ longer than the Li-O distance in Table I; this turns out to be in good agreement with the available experimental data of Kameda et al. $(1.96 \AA) .{ }^{38}$ The second peak of $g_{\mathrm{LiO}}(r)$ is well separated from the first peak, which is noticeably different from the corresponding RDF of $\mathrm{Na}^{+}$and $\mathrm{K}^{+}$shown in Figs. 2(a) and 3(a), respectively. As far as sodium is concerned, we remark that the position of the first peak $(2.40 \AA$, about $0.11 \AA$ longer than the Na-O distance in Table I) of $g_{\mathrm{NaO}}(r)$ matches very well the experimental data of Skipper and Neilson ${ }^{39}$ (2.39 ̊), and Kameda et al. $^{40}(2.40 \AA)$.

In the case of potassium, as shown in Fig. 3(a), the first peak of $g_{\mathrm{KO}}(r)$ centered at $2.85 \AA$ (about $0.2 \AA$ longer than the $\mathrm{K}-\mathrm{O}$ distance in Table $\mathrm{I}$ ) is affected by a considerable broadening compared to the corresponding peak of $g_{\mathrm{LiO}}(r)$ and $g_{\mathrm{NaO}}(r)$. Both X-ray and neutron diffraction experiments ${ }^{41,42}$ for $\mathrm{KCl}$ aqueous solutions give a potassiumoxygen distance of $2.8 \AA$ in rather good agreement with our theoretical result. Note that the first peak of $g_{\mathrm{KO}}(r)$ is located at almost the same position as the first peak of $g_{\mathrm{OO}}(r)$ shown in Fig. 3(c). As mentioned in the introduction, this coincidence represents a serious obstacle in extracting detailed information on the hydration of $\mathrm{K}^{+}$from the experimental diffraction data. In this respect, simulations play a crucial role in disentangling the two contributions.

The coordination number $N_{\text {coord }}$ of $\mathrm{Li}^{+}$with $\mathrm{H}_{2} \mathrm{O}$ molecules forming its first solvation shell does not undergo noticeable fluctuations, which originates from the well defined first peak of the corresponding RDF. As a consequence, $N_{\text {coord }}$ takes the unambiguous value of 4.0. On the contrary, the determination of $N_{\text {coord }}$ for the other two ions from the integrated radial distribution functions is more problematic due to the lack of a clear plateau in the running coordination number of $\mathrm{Na}^{+}$and $\mathrm{K}^{+}$with oxygen atoms, shown as dashed lines in Figs. 2(a) and 3(a), respectively. In an attempt at removing such ambiguities, hydration water molecules are identified in this work by adopting three different geometrical cut-off distances between the ion and water oxygen for $\mathrm{Na}^{+}$and $\mathrm{K}^{+}$. The cutoff distances used were 3.1, 3.2, and 3.3 $\AA$ for $\mathrm{Na}^{+}$and 3.6, 3.7, and $3.8 \AA$ for $\mathrm{K}^{+}$. As can be inferred from Figs. 2(a) and 3(a), these values belong to the range in which $g_{\mathrm{NaO}}(r)$ and $g_{\mathrm{KO}}(r)$ have their first minimum. The corresponding distributions of the instantaneous coordination
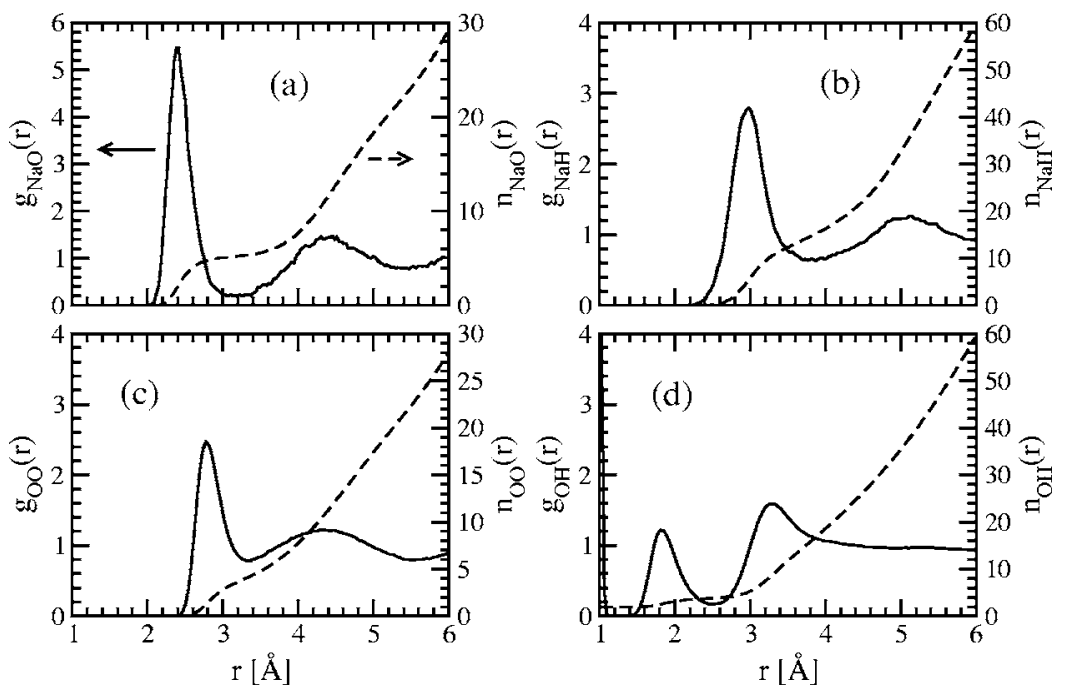

FIG. 2. Radial distribution functions (a) $g_{\mathrm{NaO}}(r)$, (b) $g_{\mathrm{NaH}}(r)$, (c) $g_{\mathrm{OO}}(r)$, and (d) $g_{\mathrm{OH}}(r)$ for $\mathrm{Na}^{+}$in liquid water. The dashed line in each panel represents the corresponding running coordination number $n(r)$. 

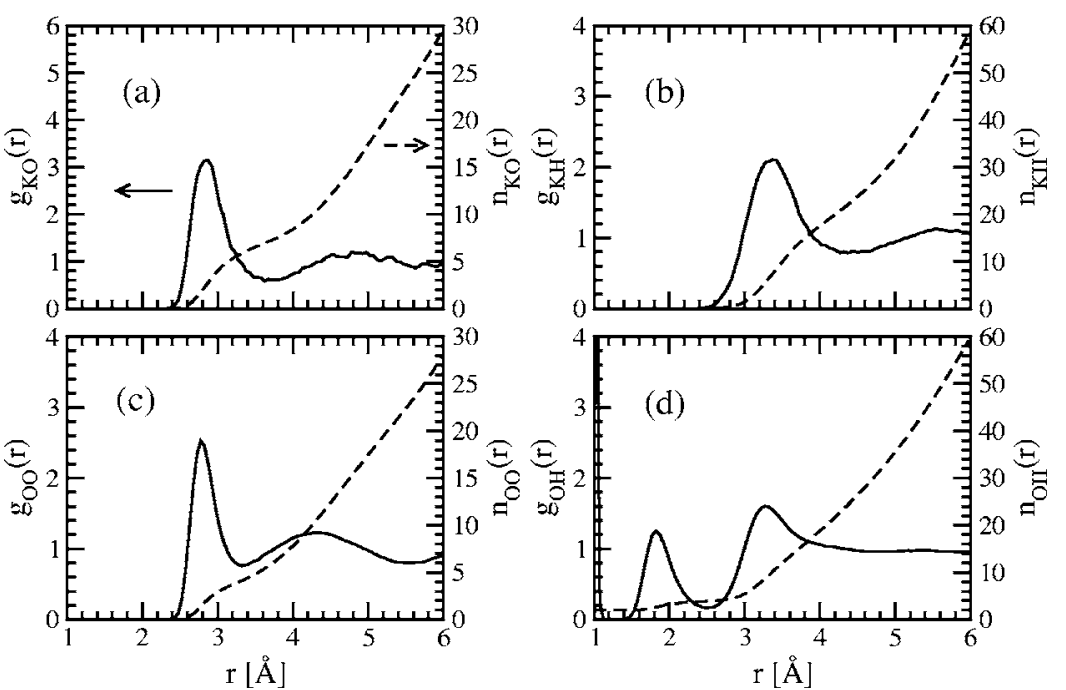

FIG. 3. Radial distribution functions (a) $g_{\mathrm{KO}}(r)$, (b) $g_{\mathrm{KH}}(r),(\mathrm{c}) g_{\mathrm{OO}}(r)$, and (d) $g_{\mathrm{OH}}(r)$ for $\mathrm{K}^{+}$in liquid water. The dashed line in each panel represents the corresponding running coordination number $n(r)$. numbers $n$ for $\mathrm{Na}^{+}$and $\mathrm{K}^{+}$are shown in Fig. 4. It is quite evident that the distribution of the instantaneous coordination number for $\mathrm{K}^{+}$is much more sensitive to the geometrical criteria adopted than that for $\mathrm{Na}^{+}$. Therefore, $\mathrm{K}^{+}$requires special care in order to draw any sound conclusion about its hydration properties. The coordination number of $\mathrm{Na}^{+}$was computed by statistical averaging on the trajectory; the value estimated in this way turns out to be $5.2 \pm 0.1$ in good agreement with $\mathrm{X}$-ray data. ${ }^{39}$ The error bar \pm 0.1 corresponds to the different cut-off distances of 3.1, 3.2, and 3.3 $\AA$. Similar procedure gives $7.1 \pm 0.4$ as the coordination number for $\mathrm{K}^{+}$. Note that our estimated coordination number for $\mathrm{Na}^{+}$is smaller than that obtained by the majority of the classical simulations $(\sim 6.0){ }^{43}$ This discrepancy indicates rather clearly that most of the present force fields used in classical molecular simulations are insufficient to describe the hydration of simple metal cations.

A typical average hydration structure of $\mathrm{Na}^{+}$and $\mathrm{K}^{+}$in the dominant coordination is shown in Fig. 5 along with the rather rigid hydration structure of $\mathrm{Li}^{+}$. As shown in Figs. 5(a) and 5(b) the hydration structure of $\mathrm{Li}^{+}$and $\mathrm{Na}^{+}$can be assigned, respectively, as tetrahedral and distorted trigonal bipyramid (or square pyramidal) structure, while for $\mathrm{K}^{+}$the hydration structure cannot be characterized by a definite geometry. This observation suggests that the hydration of $\mathrm{K}^{+}$ may be much more sensitive to the environment surrounding the ion than that of the other two cations.

The first solvation shell of water molecules around $\mathrm{Li}^{+}$is rather rigid and is preserved at least over the $40 \mathrm{ps}$ time scale of our simulation. Nonetheless, these hydration waters cannot be distinguished from other water molecules of the sol- vent by a simple inspection of the intramolecular structure. Figure 6 shows the distribution of the $\mathrm{OH}$ bond length $r_{\mathrm{OH}}$ and $\mathrm{HOH}$ bond angle $\theta_{\mathrm{HOH}}$ of hydration water molecules for $\mathrm{Li}^{+}$(top panels), $\mathrm{Na}^{+}$(middle panels), and $\mathrm{K}^{+}$(bottom panels). The water molecules composing the first solvation shell show basically the same distribution for both the $\mathrm{OH}$ bond length and the $\mathrm{HOH}$ bond angle as the rest of the solvent. The rather tiny differences that can be observed are in sharp contrast with the case of multivalent cations. For instance, a small multivalent cation such as $\mathrm{Al}^{3+}$ displays a clear difference in $r_{\mathrm{OH}}$ and $\theta_{\mathrm{HOH}}$ between $\mathrm{H}_{2} \mathrm{O}$ molecules in the first solvation shell and bulk water. ${ }^{13}$

A further insight is offered by the direction of the molecular dipole moments. Namely, the tilt angle $\psi$ between the dipole vector of an $\mathrm{H}_{2} \mathrm{O}$ molecule and an ideal reference vector pointing from the water oxygen to the metal cationsketched in Fig. 7-is determined by a delicate balance between the ion-water interactions and the hydrogen bonds formed by water molecules belonging to the first and second solvation shells. The tilt angle $\psi$ is found to take on average $141^{\circ}, 134^{\circ}$, and $124^{\circ}$ for $\mathrm{Li}^{+}, \mathrm{Na}^{+}$, and $\mathrm{K}^{+}$, respectively, thus showing a clear tendency for the tilt angle $\psi$ to decrease from $\mathrm{Li}^{+}$to $\mathrm{K}^{+}$. A large amount of hydration water molecules having $\psi<90^{\circ}$ are also observed for $\mathrm{K}^{+}$. The rather large tilting allows hydration water molecules to form three hydrogen bonds (H-bonds) per molecule with nearby $\mathrm{H}_{2} \mathrm{O}$ monomers, as will be discussed in more detail in Sec. III C. One of these $\mathrm{H}$-bonds is of the acceptor type, while the remaining two are donor type.

All the structural properties discussed above corroborate the general picture of a weaker hydration for $\mathrm{K}^{+}$. We can
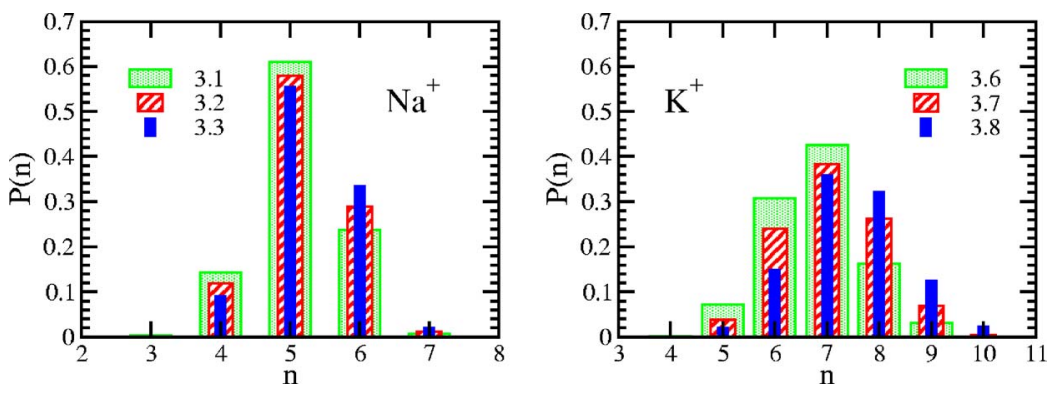

FIG. 4. Dependence of the instantaneous coordination number $n$ for $\mathrm{Na}^{+}$(left) and $\mathrm{K}^{+}$(right) on the geometrical cutoff distance. 


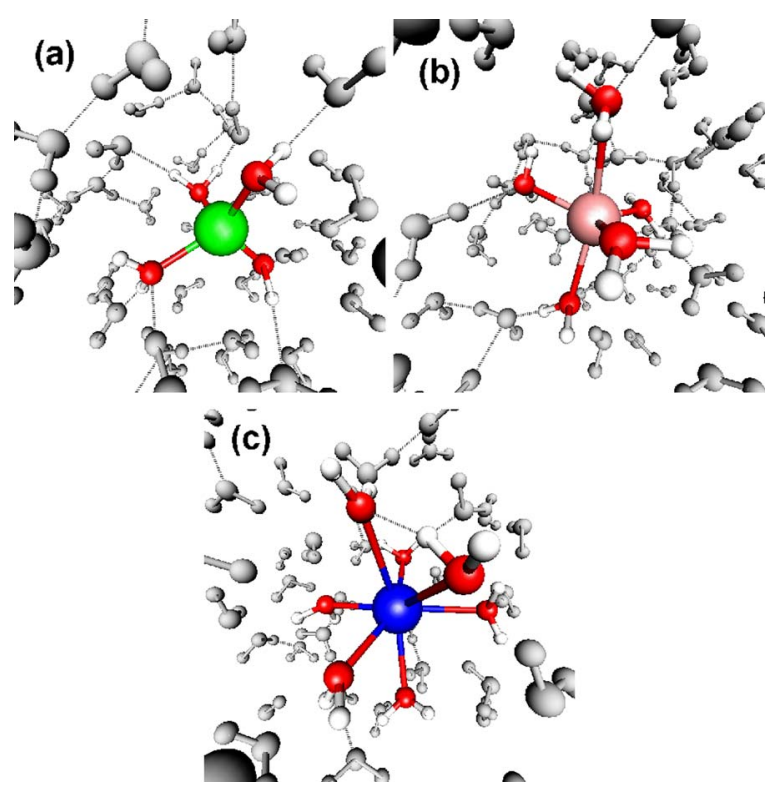

FIG. 5. Typical hydration structure for (a) $\mathrm{Li}^{+}$, (b) $\mathrm{Na}^{+}$, and (c) $\mathrm{K}^{+}$. The hydration structure of $\mathrm{Li}^{+}$and $\mathrm{Na}^{+}$shown in (a) and (b) can be assigned as tetrahedral and distorted trigonal bipyramid (or square pyramidal) structure, respectively. The hydration structure of $\mathrm{K}^{+}$cannot be characterized by a definite geometry.

then infer that, from an energetics point of view, the ionwater interactions for $\mathrm{K}^{+}$are competitive with the H-bond formation as opposed to the stronger interactions in the case of $\mathrm{Li}^{+}$.

\section{B. Dynamical properties}

The influence of the different hydration behavior of the alkali ions is remarkable when we consider dynamical properties, rather than the static average picture of the hydration structures. In particular, the self-diffusion coefficient and the rotational correlation time of water molecules in the presence of solute ions provide an evident influence of the solute on the behavior of the solvent.

Figure 8 shows the dependence of the mean square displacement (MSD) of $\mathrm{H}_{2} \mathrm{O}$ molecules on the ion concentration for our simulated aqueous solutions. By increasing the

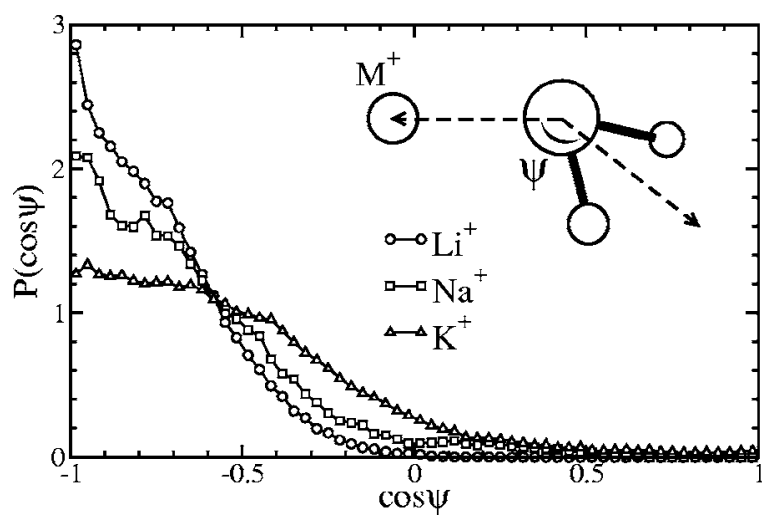

FIG. 7. Distribution of the cosine of the tilt angle $\psi$ between the dipole vector of hydration water molecule and the vector pointing from water oxygen to the metal ion. Open circles, squares, and triangles refer to the cases of $\mathrm{Li}^{+}, \mathrm{Na}^{+}$, and $\mathrm{K}^{+}$aqueous system, respectively.

ion concentration, water molecules become less diffusive in the case of $\mathrm{Li}^{+}$, at variance with $\mathrm{K}^{+}$, where their diffusivity definitely increases. The behavior of $\mathrm{Na}^{+}$in our simulation is qualitatively similar to that of $\mathrm{K}^{+}$but the concentration dependence is much weaker. In Table II the self-diffusion coefficients $D_{\text {theory }}$ estimated from the slope of the MSD for water molecules and the metal ion are reported along with the experimental data $D_{\text {exp }}$ measured on aqueous solutions of alkali chlorides at the concentration shown in parentheses. As a reference for further discussion, we have also calculated the self-diffusion coefficient of bulk water with the ordinary liquid density $\left(1.0 \mathrm{~g} / \mathrm{cm}^{3}\right)$ by performing a $20 \mathrm{ps}$ CPMD run on a $64 \mathrm{H}_{2} \mathrm{O}$ molecules system in the same simulation cell used for the alkali ion solutions and obtained $D_{\text {theory }}=1.41$ $\times 10^{-5} \mathrm{~cm}^{2} / \mathrm{s}$. ${ }^{44}$ Although our simulations underestimate the self-diffusion coefficient $D$ of water molecules by a factor of about 2, they are able to reproduce rather well the qualitative aspects despite the absence of explicit counter anions in our simulation cell. In particular, the decrease (increase) of the self-diffusion coefficient upon high concentration of $\mathrm{Li}^{+}\left(\mathrm{K}^{+}\right)$ is correctly reproduced by the simulations if compared to $D_{\text {theory }}$ for bulk water. However, we have to stress that the ion concentration dependence is likely to be overestimated pre-
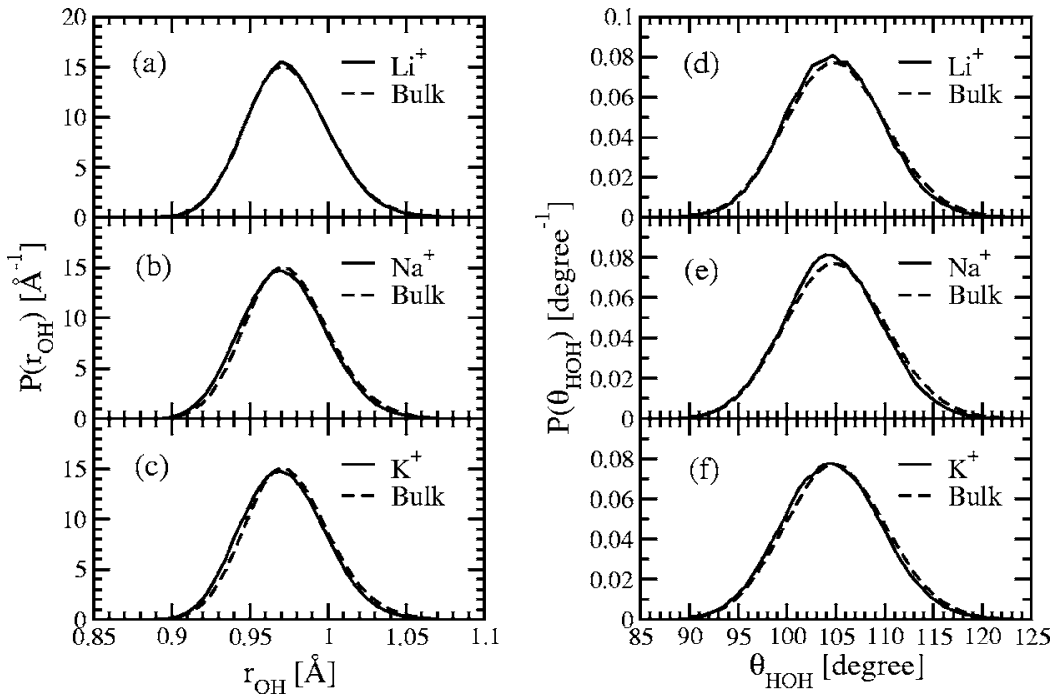

FIG. 6. Distribution of the $\mathrm{OH}$ bond length $r_{\mathrm{OH}}$ (left) and the $\mathrm{HOH}$ bond angle $\theta_{\mathrm{HOH}}$ (right) of hydration water molecules. For comparison, the corresponding distributions for the bulk are shown as dashed lines. 


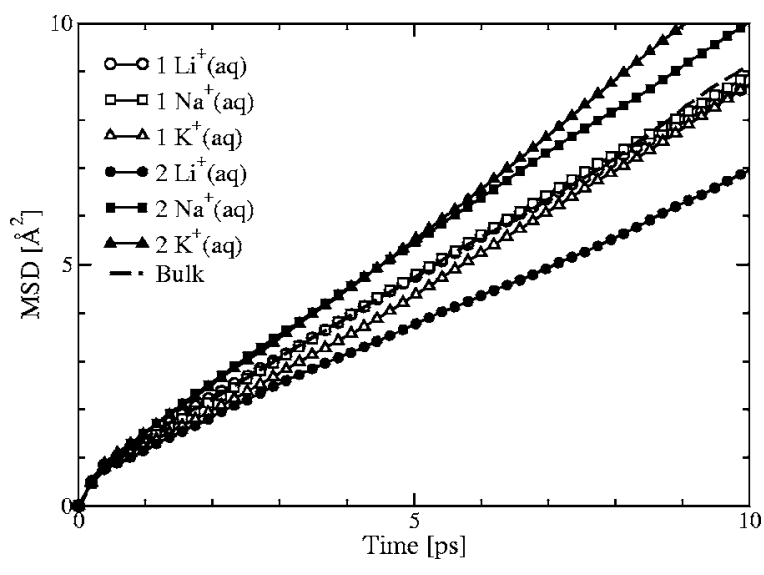

FIG. 8. Mean square displacement (MSD) for water molecules in our two systems composed of $n \mathrm{M}^{+}(\mathrm{M}=\mathrm{Li}, \mathrm{Na}$, and $\mathrm{K})$ and $(64-n) \mathrm{H}_{2} \mathrm{O},(n$ $=1,2$ ). Open circles, squares, and triangles indicate the displacement of water molecules in the system including one $\mathrm{Li}^{+}$, one $\mathrm{Na}^{+}$, and one $\mathrm{K}^{+}$ion, respectively. Filled circles, squares, and triangles represent the MSD of water molecule for the systems with two $\mathrm{Li}^{+}$, two $\mathrm{Na}^{+}$, and two $\mathrm{K}^{+}$, respectively. The reference data for bulk water, estimated from a $64 \mathrm{H}_{2} \mathrm{O}$ molecules system in the same cubic cell, are shown as dashed line.

sumably due to finite size effects induced by the small simulation box. As for the diffusion of the alkali ions, our rough estimate of the self-diffusion coefficients interestingly suggests that the diffusive motion of potassium ions is significantly enhanced at high concentrations.

The peculiar ion concentration dependence discussed above is also found in the reorientational motion of water molecules in alkali ion solutions. Figure 9 shows the orientational autocorrelation functions $C_{2}^{\mathrm{HH}}(t)=\left\langle P_{2}\left(\mathbf{e}_{i}(t) \cdot \mathbf{e}_{i}(0)\right)\right\rangle$ of solvent water molecules composing our solutions. Here, $\mathbf{e}_{i}$ is a unit vector along the $\mathrm{HH}$ vector connecting two $\mathrm{H}$ atoms of the $i$ th water molecule, and $P_{2}(\cdot)$ is the second Legendre function. Correlated with the diffusive motion, the reorientational motion of water molecules is suppressed for $\mathrm{Li}^{+}$by increasing the ion concentration, while for $\mathrm{Na}^{+}$and $\mathrm{K}^{+}$it is enhanced. The rotational correlation time $\tau_{2 \mathrm{R}}$ can be easily estimated from the decay rate of $C_{2}^{\mathrm{HH}}(t)$ and turns out to be 4.2, 4.6, and $4.8 \mathrm{ps}$ for low concentration solutions of $\mathrm{Li}^{+}$,

TABLE II. Self-diffusion coefficients $D$ of water and metal ions for our aqueous systems with one alkali ion $\left(1 \mathrm{M}^{+}\right)$and two alkali ions $\left(2 \mathrm{M}^{+}\right)$compared. The experimental values $D_{\exp }$ were taken for aqueous solutions of alkali chlorides at the concentration in unit of $\mathrm{M}$ shown in parenthesis. (One alkali ion and two alkali ions in the present simulation correspond to about 0.9 and $1.8 \mathrm{M}$, respectively.) The theoretical value $D_{\text {theory }}$ for bulk water was estimated as $1.41 \times 10^{-5} \mathrm{~cm}^{2} / \mathrm{s}$ from the slope of the mean-square displacement given in Fig. 8.

\begin{tabular}{lcccl}
\hline \hline System & Particle & \multicolumn{2}{c}{$D_{\text {theory }}\left(10^{-5} \mathrm{~cm}^{2} / \mathrm{s}\right)$} & \multicolumn{1}{c}{$D_{\text {exp }}\left(10^{-5} \mathrm{~cm}^{2} / \mathrm{s}\right)$} \\
\cline { 3 - 4 } & & $1 \mathrm{M}^{+}$ & $2 \mathrm{M}^{+}$ & \\
\hline $\mathrm{Li}^{+}(\mathrm{aq})$ & Water & 1.24 & 1.09 & $2.00^{\mathrm{a}}(2.0), 1.75^{\mathrm{a}}(3.6)$ \\
& $\mathrm{Li}^{+}$ & 0.2 & 0.6 & $0.60^{\mathrm{b}}$ \\
$\mathrm{Na}^{+}(\mathrm{aq})$ & $\mathrm{Water}^{\mathrm{N}}$ & 1.47 & 1.50 & $2.10^{\mathrm{a}}(2.0), 1.95^{\mathrm{a}}(3.6)$ \\
& $\mathrm{Na}^{+}$ & 0.3 & 1.4 & $0.91^{\mathrm{b}}$ \\
$\mathrm{K}^{+}(\mathrm{aq})$ & Water & 1.41 & 1.80 & $2.34^{\mathrm{a}}(2.0), 2.40(3.6)$ \\
& $\mathrm{K}^{+}$ & 1.0 & 2.6 & $1.10^{\mathrm{b}}$ \\
\hline \hline
\end{tabular}

${ }^{\mathrm{a}}$ Reference 56.

${ }^{\mathrm{b}}$ Reference 57.

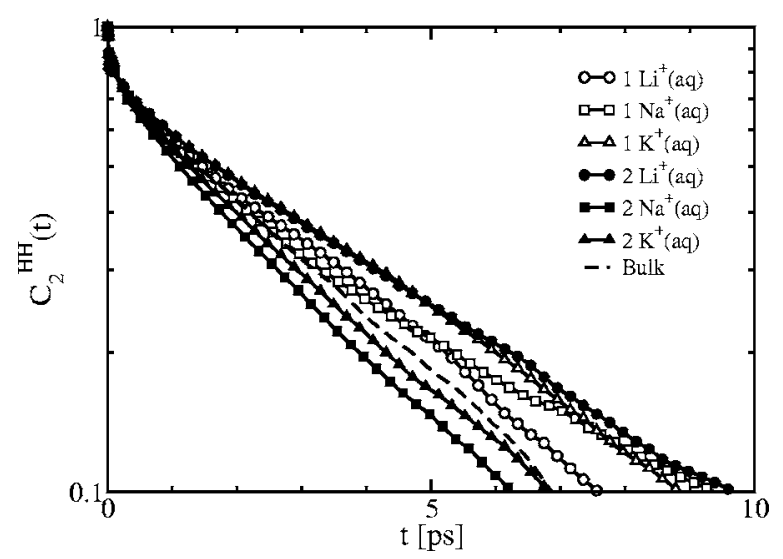

FIG. 9. Orientational autocorrelation function $C_{2}^{\mathrm{HH}}(t)$ of water molecules in the two simulated systems composed of $n \mathrm{M}^{+}(\mathrm{M}=\mathrm{Li}, \mathrm{Na}$, and $\mathrm{K})$ and $(64-n) \mathrm{H}_{2} \mathrm{O},(n=1,2)$. Open circles, squares, and triangles indicate the autocorrelation function for the system including one $\mathrm{Li}^{+}$, one $\mathrm{Na}^{+}$, and one $\mathrm{K}^{+}$ion, respectively. Filled circles, squares, and triangles are for the systems with two $\mathrm{Li}^{+}$, two $\mathrm{Na}^{+}$, and two $\mathrm{K}^{+}$, respectively. The reference data for bulk water composed of $64 \mathrm{H}_{2} \mathrm{O}$ molecules are shown as dashed line.

$\mathrm{Na}^{+}$, and $\mathrm{K}^{+}$, respectively. When we increased the concentration by adding one more metal ion, $\tau_{2 \mathrm{R}}$ changed as $4.8,3.1$, and $3.3 \mathrm{ps}$ for $\mathrm{Li}^{+}, \mathrm{Na}^{+}$, and $\mathrm{K}^{+}$, respectively. Note that the theoretical value of $\tau_{2 \mathrm{R}}$ for bulk water, estimated in exactly the same way, is $\tau_{2 \mathrm{R}}=3.5 \mathrm{ps}$, which is roughly twice the values reported in the literature. ${ }^{6}$

In accordance to the underestimation of the selfdiffusion coefficient $D$ of water molecules, the rotational correlation time $\tau_{2 \mathrm{R}}$ of water molecules is likely to be overestimated. Nonetheless, our simulations are able to capture the qualitative difference in the dynamics of the solvent $\mathrm{H}_{2} \mathrm{O}$ molecules induced by the presence of solute alkali ions. More specifically, $\mathrm{Li}^{+}$in water at ambient conditions suppresses the diffusivity and the rotational motion of water molecules, whereas $\mathrm{K}^{+}$enhances these motions. Consequently, the residence time $\tau_{\text {res }}$ of water molecules in the first shell of $\mathrm{K}^{+}\left(\tau_{\text {res }} \sim 10 \mathrm{ps}\right)$ roughly estimated by following the prescription of Impey et al. ${ }^{45}$ is much smaller than that of $\mathrm{Li}^{+}$ ( $\tau_{\text {res }}>40 \mathrm{ps}$ ). These confirm the structure-making (-breaking) effects of $\mathrm{Li}^{+}\left(\mathrm{K}^{+}\right)$postulated on the basis of experimental data. ${ }^{46}$ Our simulations also suggest that $\mathrm{Na}^{+}$ enhances the diffusive and rotational motions of water molecules in a way not too different from as $\mathrm{K}^{+}$. This is in contrast with the usual classification of $\mathrm{Na}^{+}$as a structuremaker cation. This apparent discrepancy could be mainly attributed to the following two problems in the present simulation. One is that as shown by recent extensive studies, ${ }^{19,20,23}$ the first principles MD based on the present GGA level of theory gives the slightly overstructured oxygen-oxygen correlation function and slow dynamics of water molecules compared to experiment (see Table II). This aspect may bias metal ions toward structure breakers. Another is the absence of anions in our simulation systems. Anions will affect the water dynamics and also may modify the role of cations. Inclusion of anions in the simulation will be an important future subject. 

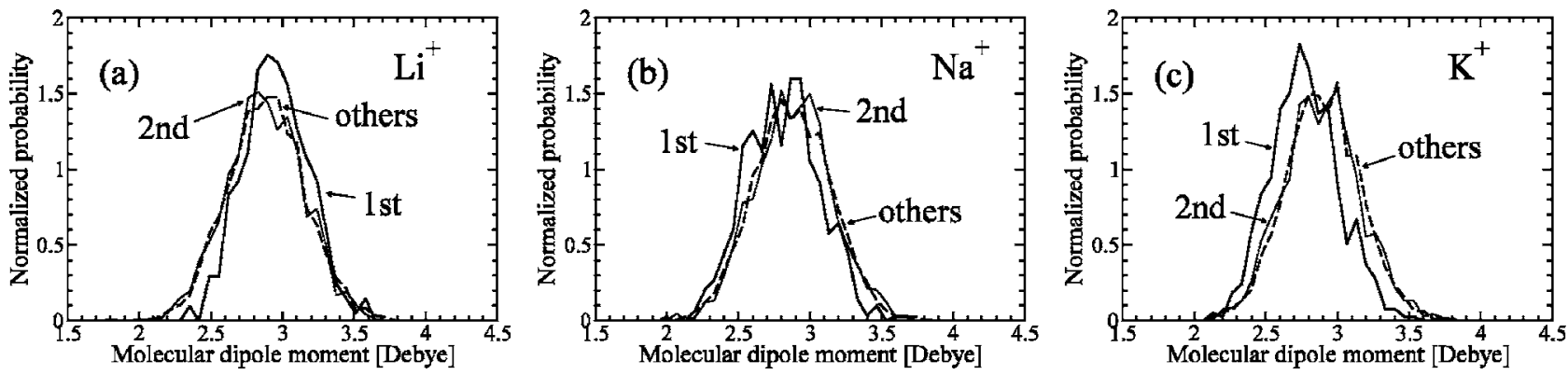

FIG. 10. Distribution of molecular dipole moments of waters calculated from maximally localized Wannier function centers for (a) $\mathrm{Li}^{+}$, (b) $\mathrm{Na}^{+}$, and (c) $\mathrm{K}^{+}$ aqueous solutions. The water molecules contained in our systems are classified into the hydration water molecules in the first shell, in the second shell, and the others intended as the rest of the solvent.

\section{Electronic properties}

It is well known that the electric dipole moment of water molecules is sensitive to the environment ${ }^{21,47-49}$; experimentally, the molecular dipole moment $\mu$ of $\mathrm{H}_{2} \mathrm{O}$ is $1.85 \mathrm{D}$ in the gas phase $^{50}$ and $\mu=2.9 \pm 0.6 \mathrm{D}$ in the normal liquid state. ${ }^{51}$ This trend holds also for hydration water molecules as demonstrated in the recent theoretical studies for various aqueous solutions. ${ }^{11,12,14,52}$ In this respect, first principles simulations have been able to reproduce the variations of $\mu$, whereas classical polarizable force fields are often inadequate. ${ }^{53}$ Figure 10 shows the distribution of the molecular dipole moment of water calculated by means of maximally localized Wannier function centers (WFC $)^{47,54}$ for our aqueous solution containing one alkali ion. In Fig. 10 the water molecules contained in our systems are classified into three groups: the hydration water molecules in the first solvation shell, the ones in the second shell, ${ }^{55}$ and the others. It turns out that only those water molecules that belong to the first solvation shell have such a dipole moment as is generally different from the rest of solvent. The average value of $\mu$ of water molecules in the first shell results in 2.96, 2.81, and 2.77 D for $\mathrm{Li}^{+}, \mathrm{Na}^{+}$, and $\mathrm{K}^{+}$, respectively. On the other hand, the rest of water molecules in all our simulations keep basically the same dipole moment of 2.89, 2.88, and $2.90 \mathrm{D}$ for $\mathrm{Li}^{+}, \mathrm{Na}^{+}$, and $\mathrm{K}^{+}$, respectively. Thus, the dipole moment of water molecules forming the first solvation shell of the alkali ions certainly show the tendency to decrease going from $\mathrm{Li}^{+}$to $\mathrm{K}^{+}$.

Analyses of our simulations reveal that the dipole moment of water molecules in the first shell of $\mathrm{Na}^{+}$and $\mathrm{K}^{+}$ decreases by $\sim 0.1 \mathrm{D}$ compared to that in the bulk liquid water. This prominent feature arises from the charge redistribution on the oxygen atoms of hydration $\mathrm{H}_{2} \mathrm{O}$ molecules. This charge redistribution phenomenon can be visualized in terms of the shift of the WFCs. In the left panel of Fig. 11 we compare the distribution of the O-WFC distance $r_{\mathrm{O}-\mathrm{WFC}}$ for water molecules belonging to the first solvation shell of the metal ions with that in the bulk. It is rather evident that the centroid of Wannier functions corresponding to the lone-pair orbitals for the $\mathrm{Na}^{+}$and $\mathrm{K}^{+}$cases is shifted toward the oxygen side, whereas the distribution of WFCs corresponding to the bonding $\sigma$ orbitals coincides almost perfectly with that for the bulk. Let us recall here the analysis in Fig. 7 concerning the tilting of the molecular dipole vector with respect to the oxygen-metal axis. This tilt allows hydration $\mathrm{H}_{2} \mathrm{O}$ monomers to form on average three hydrogen bonds per molecule as shown schematically in Fig. 12. Consequently, only one of
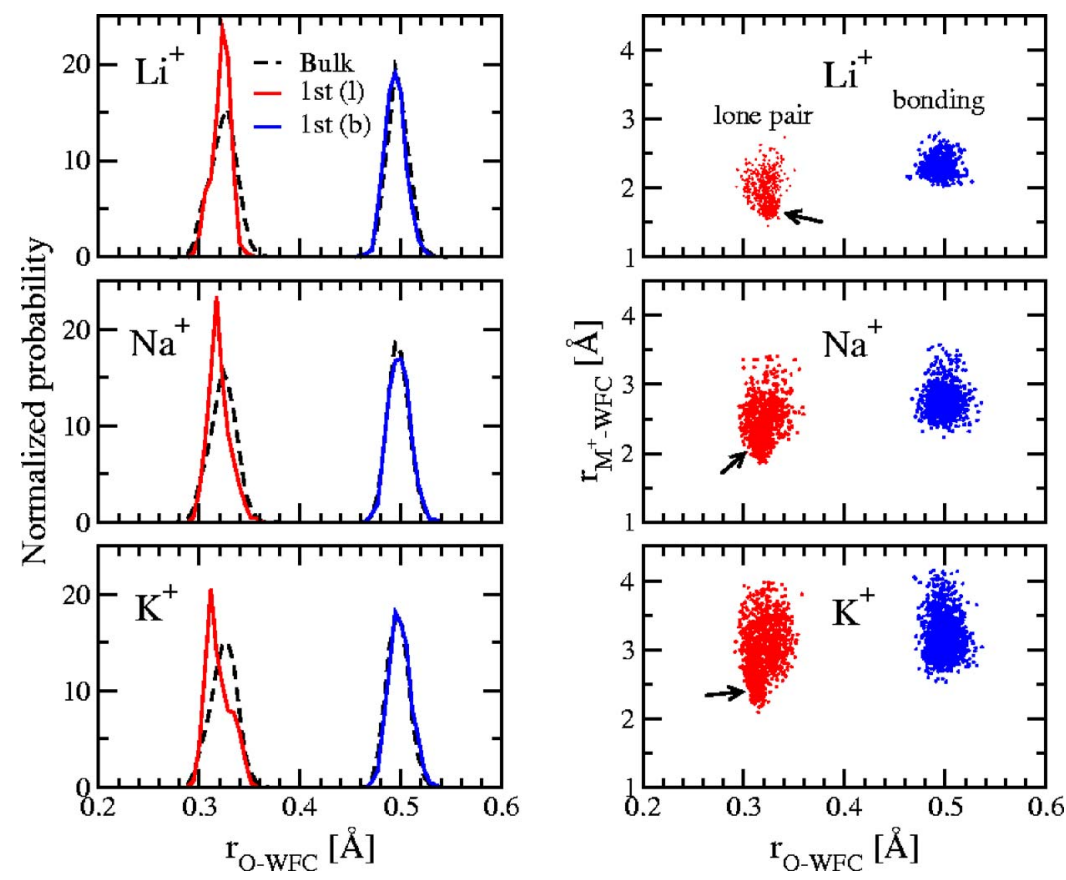

FIG. 11. (Left) Distribution of the O-WFC distance $r_{\mathrm{O}-\mathrm{WFC}}$ for water molecules in the first solvation shell of the alkali ion (solid line) and in the bulk (dashed line). The corresponding scatter graphs in the plane of ( $\left.r_{\mathrm{O}-\mathrm{WFC}}, r_{\mathrm{M}^{+}-\mathrm{WFC}}\right)$ are shown in right panel, where $\mathrm{M}$ $=\mathrm{Li}, \mathrm{Na}$, and $\mathrm{K}$. A data accumulation leading to a high density in the distribution is seen in the regions marked with arrows. 


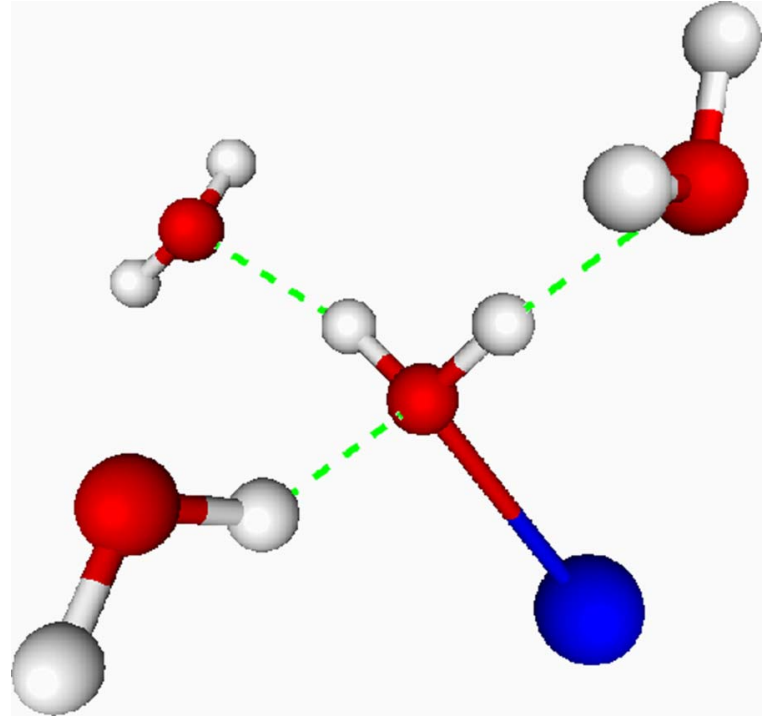

FIG. 12. Schematic structure of hydrogen bond network formed around a hydration water molecule of the alkali ion. Hydrogen bonds are indicated by dashed lines.

the lone pairs has a significant influence on (or is influenced by) the alkali ion. Hence, the water lone-pair orbital directed toward the metal ion results to be contracted by the weaker interaction than a normal H-bond typical of bulk water. This picture emerges from the distribution of WFCs mapped onto the space spanned by the two distances $r_{\mathrm{O}-\mathrm{WFC}}=\mid \mathbf{R}_{\mathrm{O}}$ $-\mathbf{R}_{\mathrm{WFC}} \mid$ and $r_{\mathrm{M}^{+}-\mathrm{WFC}}=\left|\mathbf{R}_{\mathrm{M}^{+}}-\mathbf{R}_{\mathrm{WFC}}\right|$ shown in the right panel of Fig. 11. As a matter of fact, data points of WFC are observed to be more densely accumulated at around the specific point to each alkali ion marked with arrows. These specific points are found to be located almost on the line connecting the alkali ion with water oxygen.

\section{CONCLUSIONS}

The present study investigated the hydration of $\mathrm{Li}^{+}, \mathrm{Na}^{+}$, and $\mathrm{K}^{+}$in ambient liquid water via first principles molecular dynamics. Our simulations were able to reproduce the available experimental data regarding the structural properties of the solvation. A geometrically well defined hydration structure could be assigned to $\mathrm{Li}^{+}$and $\mathrm{Na}^{+}$, leading to tetrahedral and distorted trigonal bipyramidal (or square pyramidal) structure, respectively. On the other hand, the hydration of $\mathrm{K}^{+}$seems more floppy than that of the former cases and a definite geometry could not be found. Furthermore, we evidenced that the large tilting of the dipole vector of hydration water molecules with respect to the oxygen-metal axis enables hydration water molecules to form on average three hydrogen bonds per molecule. As a consequence, one of lone pair orbitals pointing at the metal cation displays a significant interaction with the alkali ion, thus inducing a dependence of the dipole moment of hydration water molecules on the solvated ionic species. Indeed, the dipole $\mu$ of hydration water in the first shell of $\mathrm{Li}^{+}, \mathrm{Na}^{+}$, and $\mathrm{K}^{+}$turned out to be $2.96,2.81$, and $2.77 \mathrm{D}$, respectively. This represents a nonnegligible difference between the average molecular dipole moment of an $\mathrm{H}_{2} \mathrm{O}$ monomer belonging to the solvation shell of the alkali ions and a molecule in a regular $\mathrm{H}$-bond network in liquid water.

Dynamical properties, given in terms of self-diffusion coefficient and rotational correlation time for the case of one and two ions in solution, provide interesting insights into the interplay between solute and solvent and how the two influence each other. Finally, the present study evidences close relationships among molecular orientation, formation of $\mathrm{H}$ bonds, and polarization of water molecules.

A complementary message that can be extracted from this set of simulations is that more accurate force fields are required to improve the reliability of classical MD simulations for aqueous solutions relevant to many fields ranging from materials sciences to chemistry and biology. We believe that our findings can be used as a benchmark in this direction for future developments of classical simulations.

\section{ACKNOWLEDGMENTS}

The authors thank Dr. Etsuro Ito for encouragement during the course of the present work. M.B. gratefully acknowledges insightful discussions with Jung Mee Park, Pier Luigi Silvestrelli, Axel Kohlmeyer, and Jürg Hutter and support from Grant in Aid No. 18054004 of MEXT. The calculations reported in this paper were performed at the JAEA supercomputer facility, Science Information Processing Center, University of Tsukuba, and High Performance Computing System, Hokkaido University.

${ }^{1}$ B. E. Conway, Ionic Hydration in Chemistry and Biophysics, Studies in Physical and Theoretical Chemistry 12 (Elsevier, Amsterdam, 1981).

${ }^{2}$ Y. Marcus, Ion Solvation (Wiley, Chichester, 1986).

${ }^{3}$ J. Burgess, Metal Ions in Solution (Ellis Horwood, New York, 1978).

${ }^{4}$ D. T. Richens, The Chemistry of Aqua Ions (Wiley, Chichester, 1997).

${ }^{5}$ Y. Marcus, Chem. Rev. (Washington, D.C.) 88, 1475 (1988).

${ }^{6}$ H. Ohtaki and T. Radnai, Chem. Rev. (Washington, D.C.) 93, 1157 (1993).

${ }^{7}$ W. Stumm and J. J. Morgan, Aquatic Chemistry, 3rd ed. (Wiley, New York, 1996).

${ }^{8}$ R. Car and M. Parrinello, Phys. Rev. Lett. 55, 2471 (1985).

${ }^{9}$ See, e.g., the monographic Parrinello Festschrift issue of ChemPhysChem 6 (2005).

${ }^{10}$ W. Kohn and L. J. Sham, Phys. Rev. 140, A1133 (1965).

${ }^{11}$ D. Marx, M. Sprik, and M. Parrinello, Chem. Phys. Lett. 273, 360 (1997).

${ }^{12}$ F. C. Lightstone, E. Schwegler, R. Q. Hood, F. Gygi, and G. Galli, Chem. Phys. Lett. 343, 549 (2001).

${ }^{13}$ T. Ikeda, M. Hirata, and T. Kimura, J. Chem. Phys. 119, 12386 (2003).

${ }^{14}$ I. Bakó, J. Hutter, and G. Pálinkás, J. Chem. Phys. 117, 9838 (2002).

${ }^{15}$ A. P. Lyubartsev, K. Laasonen, and A. Laaksonen, J. Chem. Phys. 114, 3120 (2001).

${ }^{16}$ J. A. White, E. Schwegler, G. Galli, and F. Gygi, J. Chem. Phys. 113, 4668 (2000).

${ }^{17}$ R. Vuilleumier and M. Sprik, J. Chem. Phys. 115, 3454 (2001).

${ }^{18}$ L. M. Ramaniah, M. Bernasconi, and M. Parrinello, J. Chem. Phys. 111, 1587 (1999).

${ }^{19}$ J. C. Grossman, E. Schwegler, E. W. Draeger, F. Gygi, and G. Galli, J. Chem. Phys. 120, 300 (2004).

${ }^{20}$ E. Schwegler, J. C. Grossman, F. Gygi, and G. Galli, J. Chem. Phys. 121, 5400 (2004)

${ }^{21}$ M. Boero, K. Terakura, T. Ikeshoji, C. C. Liew, and M. Parrinello, Phys. Rev. Lett. 85, 3245 (2000).

${ }^{22}$ M. Boero, T. Ikeshoji, and K. Terakura, ChemPhysChem 6, 1775 (2005).

${ }^{23}$ J. VandeVondele, F. Mohamed, M. Krack, J. Hutter, M. Sprik, and M. Parrinello, J. Chem. Phys. 122, 014515 (2005).

${ }^{24}$ F. A. Hamprecht, A. J. Cohen, D. J. Tozer, and N. C. Handy, J. Chem. Phys. 109, 6264 (1998).

${ }^{25}$ A. D. Boese, N. L. Doltisinis, N. C. Handy, and M. Sprik, J. Chem. Phys. 
112, 1670 (2000)

${ }^{26}$ N. L. Doltsinis and M. Sprik, Phys. Chem. Chem. Phys. 5, 2612 (2003).

${ }^{27}$ M. Boero, M. Tateno, K. Terakura, and A. Oshiyama, J. Chem. Theory Comput. 1, 925 (2005).

${ }^{28}$ N. Troullier and J. L. Martins, Phys. Rev. B 43, 1993 (1991).

${ }^{29}$ S. Goedecker, M. Teter, and J. Hutter, Phys. Rev. B 54, 1703 (1996).

${ }^{30}$ C. Hartwigsen, S. Goedecker, and J. Hutter, Phys. Rev. B 58, 3641 (1998).

${ }^{31}$ M. Sprik, J. Hutter, and M. Parrinello, J. Chem. Phys. 105, 1142 (1996).

${ }^{32}$ M. J. Frisch, G. W. Trucks, H. B. Schlegel et al.,Gaussian 03, Revision B.05 (Gaussian, Inc., Pittsburgh, PA, 2003).

${ }^{33}$ F. A. Bornemann and C. Schütte, Numer. Math. 78, 359 (1998).

${ }^{34} \mathrm{We}$ also noticed that our first set of $\mathrm{Li}^{+}$and $\mathrm{K}^{+}$aqueous solutions shows the difference of computed pressure by about $270 \mathrm{MPa}$ on average. However, the second peak of $g_{\mathrm{OO}}(r)$ whose position is known to be very sensitive to pressure [see, e.g., Th. Strässle et al., Phys. Rev. Lett. 96, 067801 (2006)] represents as seen in Figs. 1(c) and 3(c) almost no shift of the peak position, indicating that the pressure difference is expected to be, in reality, not so large.

${ }^{35}$ S. Nosé, J. Chem. Phys. 81, 511 (1984).

${ }^{36}$ W. G. Hoover, Phys. Rev. A 31, 1695 (1985).

${ }^{37}$ CPMD, Copyright IBM Corp. 1990-2004, Copyright MPI für Festkörperforshung Stuttgart 1997-2001.

${ }^{38}$ Y. Kameda and O. Uemura, Bull. Chem. Soc. Jpn. 66, 384 (1993).

${ }^{39}$ N. T. Skipper and G. W. Neilson, J. Phys. Condens. Matter 1, 4141 (1989).

${ }^{40}$ Y. Kameda, K. Sugawara, T. Usuki, and O. Uemura, Bull. Chem. Soc. Jpn. 71, 2769 (1998)

${ }^{41}$ N. Ohtomo and K. Arakawa, Bull. Chem. Soc. Jpn. 53, 1789 (1980).
${ }^{42}$ G. W. Neilson and G. W. Skipper, Chem. Phys. Lett. 114, 35 (1985).

${ }^{43}$ A. Tongraar, K. R. Liedl, and B. M. Rode, J. Phys. Chem. A 102, 10340 (1998).

${ }^{44}$ E. H. Hardy, A. Zygar, M. D. Zeidler, M. Holz, and F. D. Sacher, J. Chem. Phys. 114, 3174 (2001).

${ }^{45}$ R. W. Impey, P. A. Madden, and I. R. McDonald, J. Phys. Chem. 87, 5071 (1983).

${ }^{46}$ H. G. Hertz, Water, A Comprehensive Treatise (Plenum, New York, 1973).

${ }^{47}$ P. L. Silvestrelli and M. Parrinello, Phys. Rev. Lett. 82, 3308 (1999).

${ }^{48}$ J. K. Gregory, D. C. Clary, K. Liu, M. G. Brown, and R. J. Saykally, Science 275, 814 (1997).

${ }^{49}$ D. L. Bergman, Chem. Phys. 253, 267 (2000).

${ }^{50}$ S. A. Clough, Y. Beers, G. P. Klein, and L. S. Rothman, J. Chem. Phys. 59, 2254 (1973).

${ }^{51}$ Y. S. Badyal, M.-L. Saboungi, D. L. Price, S. D. Shastri, D. R. Haeffner, and A. K. Soper, J. Chem. Phys. 112, 9206 (2000).

${ }^{52}$ T. Ikeda, M. Hirata, and T. Kimura, J. Chem. Phys. 122, 024510 (2005).

${ }^{53}$ M. Boero, K. Terakura, T. Ikeshoji, C. C. Liew, and M. Parrinello, J. Chem. Phys. 115, 2219 (2001).

${ }^{54}$ N. Marzari and D. Vanderbilt, Phys. Rev. B 56, 12847 (1997).

${ }^{55}$ The second shell corresponds to the second peak of RDF. More quantitatively, it is defined as the region given by $3.0 \AA<r<5.0 \AA$ for $\mathrm{Li}^{+}$, $3.1 \AA<r<5.3 \AA$ for $\mathrm{Na}^{+}$, and $3.7 \AA<r<6.0 \AA$ for $\mathrm{K}^{+}$. The results of Fig. 10 are insensitive to the small change in the boundaries.

${ }^{56}$ K. A. Valiev and M. I. Emeliyanov, Zh. Strukt. Khim. 5, 671 (1964).

${ }^{57}$ R. A. Robinson and R. H. Stokes, Electrolyte Solutions (Butterworths, London, 1955). 